Mon. Not. R. Astron. Soc. 000, 000-000 (0000) Printed 26 October $2018 \quad$ (MN LATEX style file v1.4)

\title{
Detection of the old stellar component of the major Galactic bar
}

\author{
P. L. Hammersley ${ }^{1}$, F. Garzón ${ }^{1,2}$, T. Mahoney $^{1}$, M. López-Corredoira ${ }^{1}$, \\ M. A. P. Torres ${ }^{1}$ \\ 1 Instituto de Astrofísica de Canarias, E-38200 La Laguna, Tenerife, Spain \\ 2 Departamento de Astrofísica, Universidad de La Laguna, La Laguna, Tenerife, Spain
}

26 October 2018

\begin{abstract}
We present near-IR colour-magnitude diagrams and star counts for a number of regions along the Galactic plane. It is shown that along the $l=27^{\circ} \quad b=0^{\circ}$ line of sight there is a feature at $5.7 \pm 0.7 \mathrm{kpc}$ with a density of stars at least a factor two and probably more than a factor five times that of the disc at the same position. This feature forms a distinct clump on an $H$ vs. $J-H$ diagram and is seen at all longitudes from the bulge to about $l=28^{\circ}$, but at no longitude greater than this. The distance to the feature at $l=20^{\circ}$ is about $0.5 \mathrm{kpc}$ further than at $l=27^{\circ}$ and by $l=10^{\circ}$ it has merged with, or has become, the bulge. Given that at $l=27^{\circ}$ and $l=21^{\circ}$ there is also a clustering of very young stars, the only component that can reasonably explain what is seen is a bar with half length of around $4 \mathrm{kpc}$ and a position angle of about $43^{\circ} \pm 7^{\circ}$.
\end{abstract}

Key words: Galaxy: structure, stellar content - Infrared: stars

\section{INTRODUCTION}

'The position of the Sun in the Galactic disc makes it difficult to study the large-scale structures in the inner Galaxy. ' The interstellar extinction means that visible wavelengths cannot penetrate more than a few kpc into the plane, so until large-area IR surface brightness maps and point source , surveys became available little could be said on the distribution of stars in the inner regions. Even in the infrared there remains the problem that a single line of sight will contain sources from many Galactic components, which makes the results ambiguous. Even the Galaxy's Hubble type, of importance in understanding how the Milky Way relates to other galaxies, is still uncertain.

Recently it has become accepted that the Galaxy is a barred spiral, however there is still some considerable argument over what the bar actually is. There is general agreement that the near end of the Galactic bar is in the first quadrant, but angles between the bar and the line of sight between the Sun and the Galactic Centre (GC) vary between about $10^{\circ}$ and $75^{\circ}$. The most popular angle based on studies of the distribution of stars is between $10^{\circ}$ and $30^{\circ}$ (Dwek 1995; Freudenreich 1998; López-Corredoira et al. 2000 ). However, these studies are all based on off-plane bulge sources and so although the feature is usually referred to as the bar, it could equally well be described as a triaxial bulge. Binney et al. (1991) studied the flow of gas in the inner few degrees and also showed that the gas-flow lines followed a bar-like potential with a position angle of $16^{\circ}$.
Other authors have studied other regions in the plane and arrived at a larger angle for the bar. Sevenster et al. (1999) examined the kinematics of OH-IR stars and find a bar with an angle of $44^{\circ}$. Peters (1975) on the basis of HI maps and Nakai (1992) using large scale CO maps have both suggested a bar near $45^{\circ}$. The most extreme bar angle yet suggested is $75^{\circ}$ (Hammersley et al. 1994, hereafter Paper 1). This was based on the detection a large star formation region between $l=27^{\circ}$ and $21^{\circ}$ and then showing that a stick-like bar with a half length of about $4 \mathrm{kpc}$ would readily explain the form of the COBE surface brightness maps in the Galactic plane. Other authors, however, have preferred to ascribe these features to a ring or blobs of star formation in spiral arms or trailing from the triaxial bulge (e.g. Freudenreich 1998).

If there really were a major bar, then as well as having major SFRs at both extremes it could also have a high concentration of older stars along its whole length, if the bar is a long-lived feature. Such a clustering would form a strong giant branch in a colour-magnitude diagram for any line of sight crossing the bar. The best way to search for an old population deep within the Galactic plane is to use near-IR colour-magnitude diagrams. Infrared wavelengths penetrate the interstellar dust far more easily than optical wavelengths. Furthermore, early $\mathrm{K}$ giants, which have by far the highest space density for the giants, have a very restricted range of absolute magnitudes. This means that they naturally form a dense clump on an infrared HR diagram. 


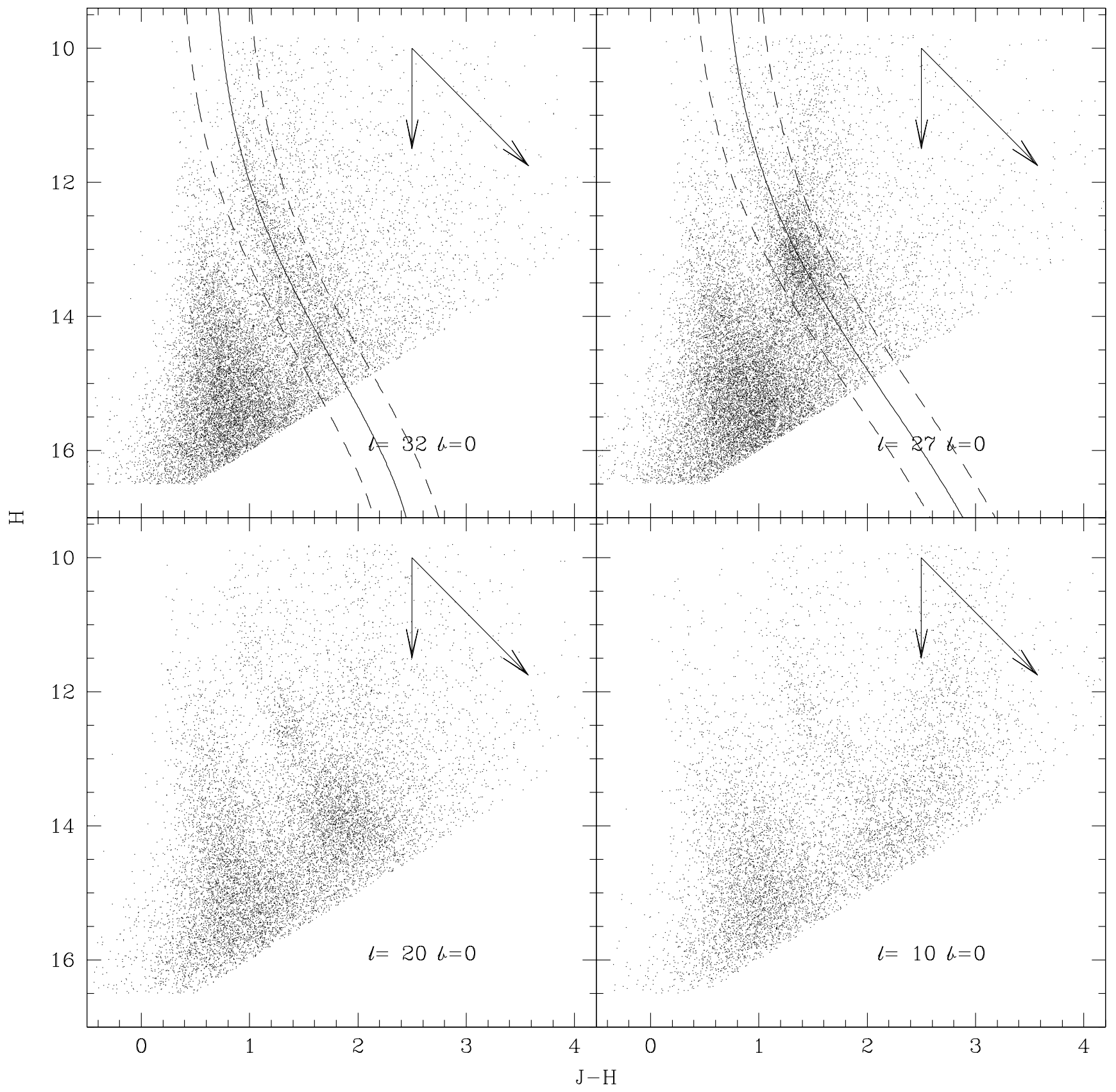

Figure 1. The $J-H$ vs. $H$ diagrams for regions on the plane at $l=32^{\circ}, 27^{\circ}, 20^{\circ}$ and $10^{\circ}$. The diagonal arrow shows 10 magnitudes of visible extinction and the vertical shows the effect of doubling the distance. The solid line going downwards from left to right on the $l=32^{\circ}$ and $27^{\circ}$ plots show the position of a K3III with increasing distance from the Sun. The two almost vertical solid lines on the $l=27^{\circ}$ plot show giant branches at $4.5 \mathrm{kpc}$ with an extinction of $A_{V}=4.7 \mathrm{mags}$ and $6.6 \mathrm{kpc}$ with $A_{V}=8.7 \mathrm{mags}$.

At the distance of interest these giants will have a $K$ magnitude of 12 to 14 making them simple to detect in a few seconds on even a small telescope.

In this paper we assume that the distance to the GC is $8 \mathrm{kpc}$, and that all positions quoted are on the Galactic plane unless otherwise stated. We will also assume absolute magnitudes for K2-3III of $M_{K}=-1.65 M_{H}=-1.5$ and $M_{J}=-0.89$ as in Table 2 of Wainscoat et al. (1992) and interstellar extinctions as given in Reike \& Lebofsky (1985) $A_{K}=0.112 A_{V}, A_{H}=0.175 A_{V}$ and $A_{J}=0.282 A_{V}$.

\section{OBSERVATIONS}

During 1999 June observations of a series of $20 \times 12$ arcmin fields were observed along the Galactic plane between $l=0^{\circ}$ and $l=37^{\circ}$, using CAIN, the facility IR camera on the $1.5 \mathrm{~m}$ TCS (Observatorio del Teide, Tenerife). The seeing was typically $1^{\prime \prime}$ and data were obtained only in photometric conditions. Star counts were then produced to $J=17, H=16.5$ and $K_{\mathrm{s}}=15.2$. Figure 1 show the $H$ vs. $J-H$ colour-magnitude diagrams for the fields on the plane at $l=32^{\circ}, 27^{\circ}, 20^{\circ}$ and $10^{\circ}$. The diagonal arrows shows the effect of 10 magnitudes of interstellar extinction in the visible. 


\section{ANALYSIS}

The main sequence stars have relatively low intrinsic luminosities, so only the closer ones are detected and there is little interstellar reddening. They form the triangular clump at $J-H=0.5$. To the right of the main sequence there is a diagonal curving stripe running from top left to bottom right. This is formed by the $\mathrm{K}$ giants in the disc with increasing distance from the Sun. The middle solid line running diagonally downwards from left to right on the $l=32^{\circ}$ and $27^{\circ}$ plots, shows the position of a K3III star on this diagram. This assumes that the extinction at any point follows a double exponential with a scale height of $50 \mathrm{pc}$ and scale length of 3500 pc, as described in Wainscoat et al. (1992). A change in absolute magnitude displaces the line vertically whereas a change in the extinction changes the slope of the line in the diagram. Therefore, the fact that this line fits the stripe means that there cannot be significant errors in the assumed absolute magnitudes and extinction. The main sequence stars and the K-giant stripe can also be clearly seen in the $l=27^{\circ}, 20^{\circ}$ and $10^{\circ}$ plots. A cluster of stars along the line of sight will form a giant branch extending upwards and slightly to the right (e.g. the bulge at $l=10^{\circ}$ at $J-H=2.8$ ). On the $l=27^{\circ}$ plot two giant branches have been plotted for distances of $4.5 \mathrm{kpc}$ with an extinction of $A_{V}=4.7 \mathrm{mags}$ and $6.6 \mathrm{kpc}$ with $A_{V}=8.7 \mathrm{mags}$.

In the middle of the $l=27^{\circ}$ stripe $\left(\mathrm{m}_{H}=13.3, J-H=1.3\right)$ there is a large clump of sources with a giant branch extending almost vertically to brighter magnitudes. This clump can also be seen at $l=20^{\circ}$ although somewhat more reddened $(J-H=2)$ and this extra extinction causes the giant branch to become less distinct. Extinction is patchy even on small angular scales and this leads to variations in the extinction to the individual sources detected. Therefore, if the average extinction rises the scatter in the magnitudes and colours will also increase. At $l=10^{\circ}$ the giant branch can be seen at $J-H=2.8$, which is due to the bulge. The distance to the bulge plus extinction is too great to see the clump of $\mathrm{K}$ giants on the stripe.

In order to gain an idea of the number of sources in the clump, the $\mathrm{K}$ giants in the $l=32^{\circ}$ and $27^{\circ}$ regions were isolated. The sources with a $J-H$ within 0.3 mag of the predicted K3III line were extracted (the dashed lines in Fig. 1) and the $K_{\mathrm{s}}$ star counts for only these source plotted in Figure 2. $K_{\mathrm{s}}$ was used as it is less effected by extinction than $H$ or $J$. The sources used here have to be detected in all three bands and the limiting magnitude for this plot is actually set by $J$ and not $K_{\mathrm{s}}$, which is why the limit is around 13.5 and not 15.2. For magnitudes brighter than about $\mathrm{m}_{K_{\mathrm{s}}}=11.5$ there is little difference between the two plots but then by $\mathrm{m}_{K_{\mathrm{s}}}=12.8$ there are three times as many sources at $l=27^{\circ}$. The clump sources are extremely well defined; the FWHM is under 1 magnitude and the spread that is seen can be attributed principally to the luminosity function (LF) with a small part to the differences in extinction and errors in the photometry. This leaves very little spread due to the distance through the feature, which implies that the feature is compact along the line of sight. As the LF is not precisely known, it is impossible to determine the distance through the clump from these data alone, however it must be significantly less than $2 \mathrm{kpc}$ as alone this would lead to a spread in magnitudes of $0.4 \mathrm{mag}$.

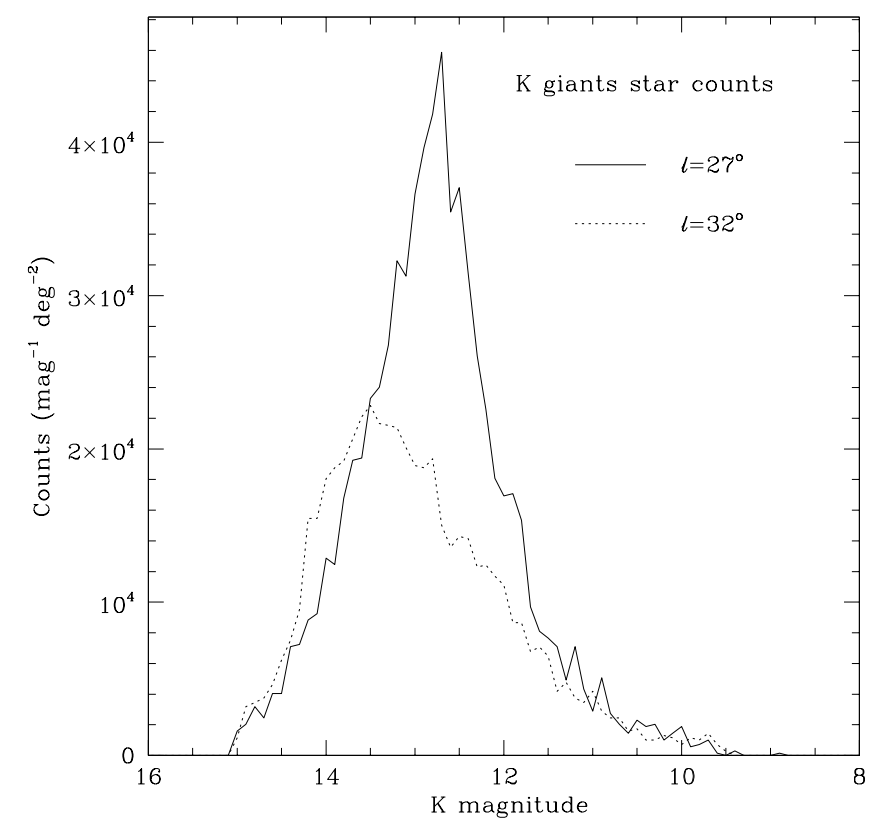

Figure 2. Differential $K_{\mathrm{s}}$ star counts for the $\mathrm{K}$ giants at $l=32^{\circ}$ and $27^{\circ}$.

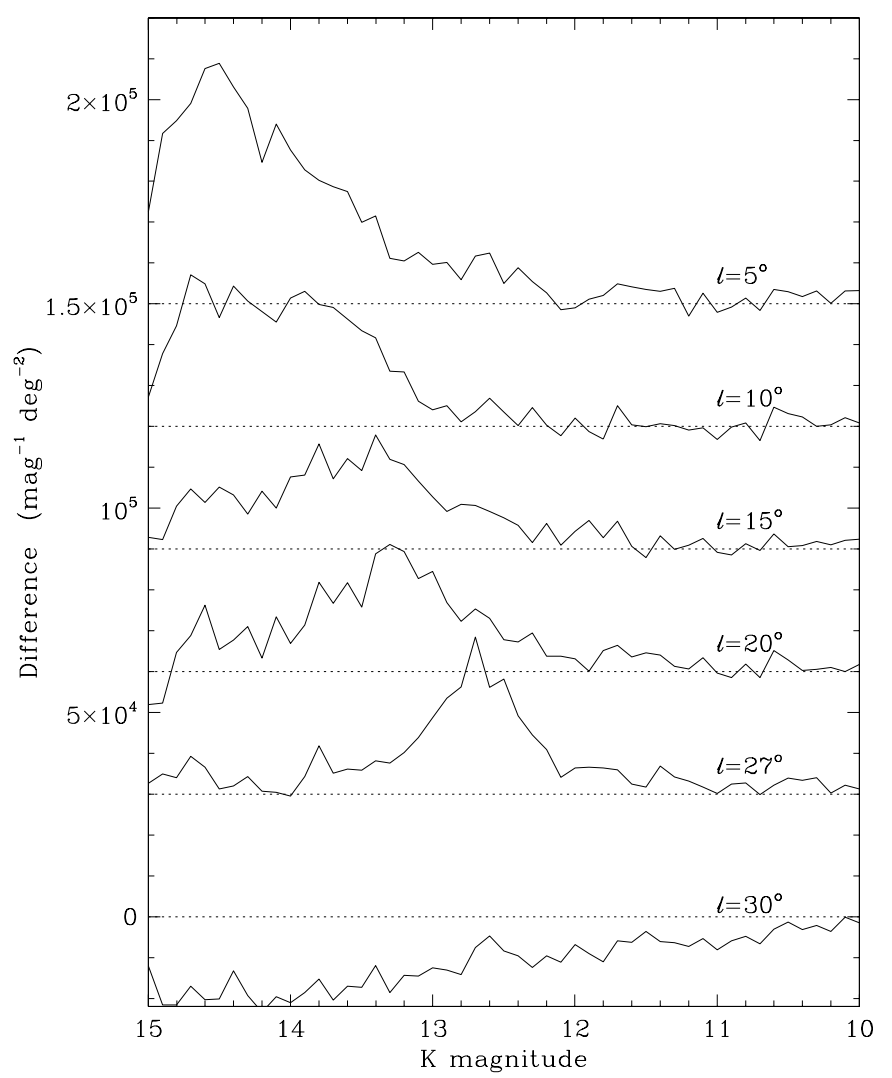

Figure 3. Differential $\mathrm{K}_{\mathrm{s}}$ star counts after removing the dwarfs and subtracting the non-dwarf counts at $l=32^{\circ}$ for the regions on the plane at $l=30^{\circ}, 27^{\circ}, 20^{\circ}, 15^{\circ}, 10^{\circ}$ and $5^{\circ}$. The curves are offset from each other by 30,000 counts and the dotted horizontal lines mark the true zero for each curve. 
Figure 3 shows the $K_{\mathrm{s}}$ differential star counts for six regions after first removing the dwarfs and then subtracting the non-dwarf counts at $l=32^{\circ}$ from the resulting counts in each region. The regions have been offset by 30,000 counts to separate the plots and the zero for each region is shown by a dotted line. The dwarfs were removed using their position on the $H$ vs. $J-H$ diagram in order to increase the contrast of any features in the inner Galaxy. There are no major clumps if the HR diagram at $l=32^{\circ}$ and the counts follow a smooth curve with magnitude (Fig. 2). By subtracting the non-dwarf $l=32^{\circ}$ counts, the steep rise in counts with increasing magnitude is reduced and the differences between the regions become far clearer. All the giants rather than just the $\mathrm{K}$ giants (as in Figure 2) were plotted as the $J$ band sensitivity is not sufficient to follow these stars to the distance of the bulge, also in many areas the $\mathrm{K}$ giant stripe becomes distorted by extinction.

The line of sight at $l=30^{\circ}$ runs into the dust lane inside the Scutum spiral arm whilst the $l=32^{\circ}$ region is tangential to the stars in the arm. Therefore, the $l=30^{\circ}$ region has more extinction than $l=32^{\circ}$ and hence the curve runs well below zero. In all of the other regions the plots are more or less zero between $\mathrm{m}_{K} \mathrm{~s}=10$ and 12 , which is surprising given the large difference in longitudes, however it does indicate that the regions can be directly compared. The peak at $l=27^{\circ}$, $\mathrm{m}_{K_{\mathrm{s}}}=12.8$ shown in Figs. 2 and 3 can be clearly seen $\left(l=27^{\circ}\right.$ was used since at $l=25^{\circ}$ there is a known dust lane which severely distorts the counts). This peak is also seen at $l=20^{\circ}$, $15^{\circ}, 10^{\circ}$ and $5^{\circ}$ but the magnitude of the peak goes fainter as the longitude decreases. At $l=27^{\circ}, 20^{\circ}$ and $15^{\circ}$ the bulge should not provide any significant counts (e.g. Freudenreich 1998; López-Corredoira et al. 2000), although by $l=10^{\circ}$ and $5^{\circ}$ the bulge is becoming important and this is seen in the increased number of sources and the increased width of the peak. Therefore, the clump at $l=27^{\circ}$ cannot belong to the bulge, but to a feature that runs into the bulge.

The $J-H$ position of the clump (Fig. 1) is at 1.32 which indicates an extinction along the line of sight to the feature at $l=27^{\circ}$ of $\mathrm{A}_{V}=6 \pm 1$ magnitudes. The peak of the $\mathrm{K}$ giants in Figure 3 is at $\mathrm{m}_{K_{\mathrm{s}}}=12.8$ and assuming an absolute magnitude of -1.65 for the $\mathrm{K}$ giants and that $A_{K_{\mathrm{s}}}=0.11 A_{V}$ gives the best distance from the Sun to the feature at $l=27^{\circ}$ of $5.7 \pm 0.7 \mathrm{kpc}$. The error is dominated by uncertainty in the absolute magnitude and extinction. The stated error was determined from the accuracy of the fit of the line to the peak of the $\mathrm{K}$ gaint stripe in Figure 1, which is 0.20 mags at the distance of the cluster. At $l=20^{\circ}$ the peak at $K_{\mathrm{s}}$ is about 0.5 mag fainter than at $l=27^{\circ}$ which, when extinction is taken into account, makes it about $0.5 \mathrm{kpc}$ further away (Figs. 1 and 3).

As cross-check for the distance to the clump at $l=27^{\circ}$ Figure 4 shows the $H$ vs. $J-H$ diagram for the region at $l=2^{\circ} \quad b=-2^{\circ}$. The bulge is clearly seen forming a giant branch extending upwards and slightly to the right near $J-$ $H=1$. However, as the line of sight runs below the Galactic plane, the extinction is far less than in Figure 1. The K giants again form a very strong clump at $\mathrm{m}_{H}=13.5, \mathrm{~J}-H$ $=0.95$. Therefore, assuming that these sources have a similar absolute magnitude as the clump at $l=27^{\circ}$, this implies that the clump at $l=27^{\circ}$ is at a distance of about 0.69 that of the bulge, i.e. $5.5 \mathrm{kpc}$. Conditions in the inner disc will be different from those in the bulge, hence this calculation can

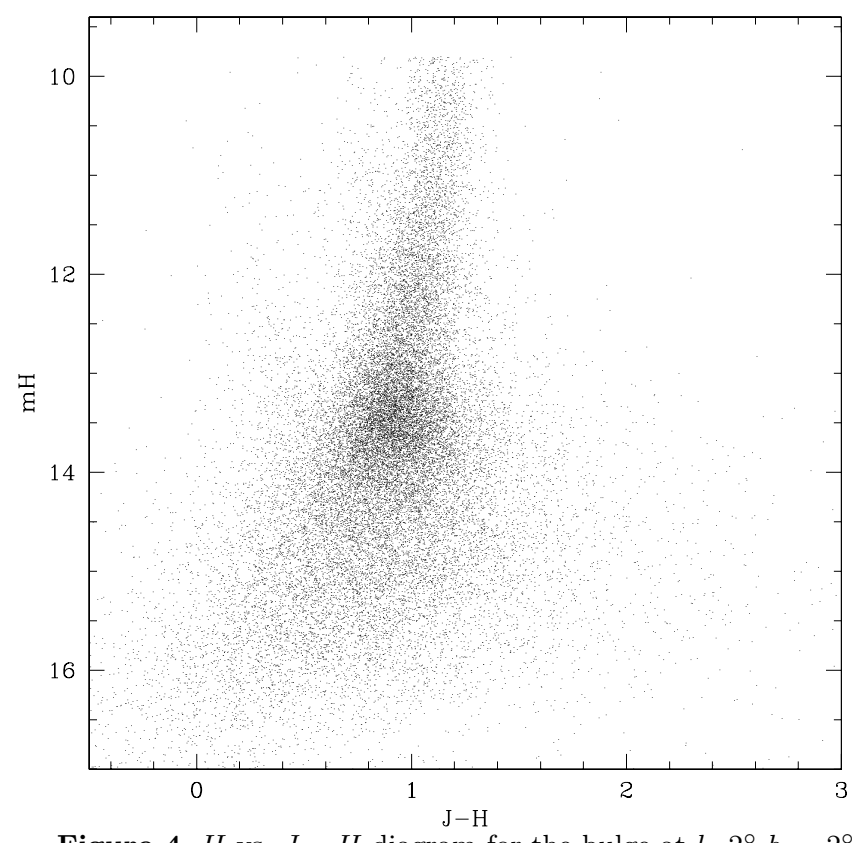

Figure 4. $H$ vs. $J-H$ diagram for the bulge at $l=2^{\circ} b=-2^{\circ}$.

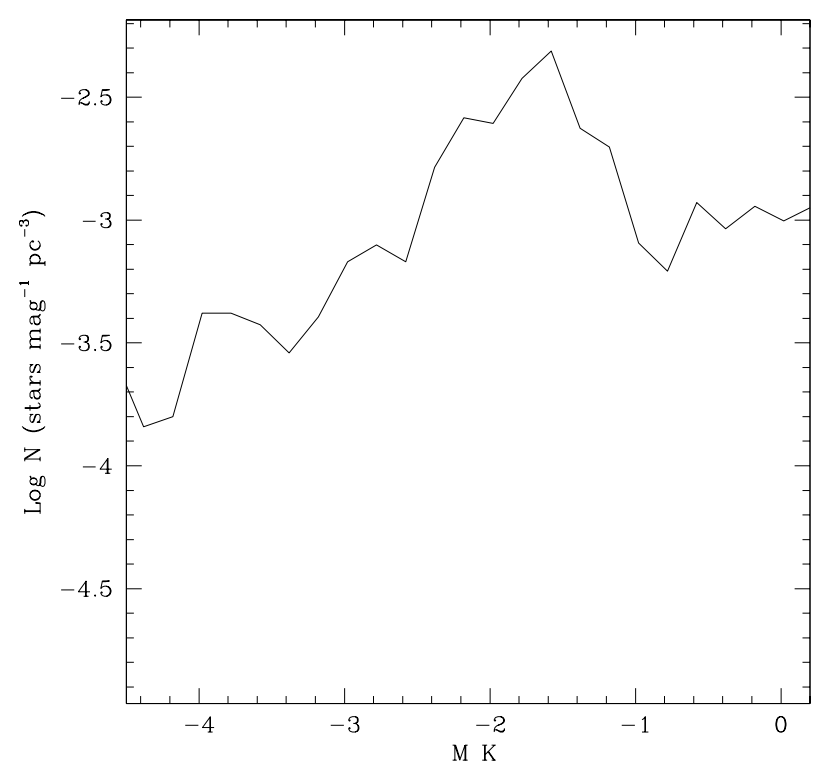

Figure 5. The K luminosity function of the clump at $l=27^{\circ}$, assuming a distance through the clump of 500pc

only be approximate but it is in agreement with the above distance.

Figure 5 gives part of the luminosity function for the clump sources in stars $\mathrm{mag}^{-1} \mathrm{pc}^{-3}$ assuming that the distance through the clump is $500 \mathrm{pc}$, if the distance turns out to be larger then the density will be proportionally less. It was obtained by isolating the sources between the giant branches shown in the $l=27^{\circ}$ plot in Figure 1 . The sources were then de-reddened assuming a distance of $5700 \mathrm{pc}$ and the standard extinction values, the apparent magnitudes were then converted into absolute magnitudes. The process was repeated for $l=32^{\circ}$ and the result was then subtracted from that for $l=27^{\circ}$ to remove the disc and leave the the 
clump sources. No attempt has been made to correct for the distance through the clump or error in the photometry. For comparison the disc at the position of the clump would have a density between 5 and 10 times lower whereas the bulge at this location would have a density over 100 times lower (e.g. Wainscoat et al., 1992, López-Corredoira et al. ,2000). The bulge reaches this sort of density at only a few hundred pc from the GC. Given that there is also a large number of very luminous young sources at $l=27^{\circ}$ (Paper 1 ), then this must be one of the most luminous and densely populated parts of the Galaxy after the GC itself.

\section{DISCUSSION}

The $K_{\mathrm{s}}$ star counts clearly show a feature where the number of old sources is similar between $l=27^{\circ}$ and $l=5^{\circ}$, but whose distance from the Sun increases with decreasing longitude. Paper 1 discusses the possible causes for the very high numbers of very luminous stars also seen at $l=27^{\circ}$ and $l=21^{\circ}$ and shows that these stars are related with the peaks in the COBE surface maps. Like the old star these young stars are seen at $l=27^{\circ}$ but not at $l=32^{\circ}$. The distance to the clump of $\mathrm{K}$ giants at $l=27^{\circ}$ is about $5.7 \mathrm{kpc}$, which is very close to the value determined in Garzón et al. (1997) for the luminous stars at $l=27^{\circ}$. This implies that there is an old population co-existing with, or very close to, a young population. Hence, the peaks in the $C O B E$ surface brightness maps near $l=27^{\circ}$, are important for understanding the structures in the inner Galaxy and should not be dismissed as patchy star formation.

Paper 1 concludes that the most probable explanation for the young stars seen at $l=27^{\circ}$ is a bar, but that a ring cannot be ruled out. The above data discounts the hypothesis that the old sources seen here belong to a ring

- A ring, which is seen tangentially at $l=27^{\circ}$, should have far more sources at $l=27^{\circ}$ than at $l=15^{\circ}$, but this is not seen.

- The ring would be significantly closer at $l=15^{\circ}$ than at $27^{\circ}$, hence the sources should appear brighter at $l=15^{\circ}$, not fainter as seen here.

A long lived bar, however, would naturally produce all of the features seen, in particular that the distance to the feature will increase with decreasing longitude if the near end of the bar is in the first quadrant.

The distance to the clump at $l=27^{\circ}$ fixes the position angle at $43^{\circ} \pm 7^{\circ}$ and a half-length of about $4 \mathrm{kpc}$. This is clearly at odds with Paper 1 , which gives an angle of $75^{\circ}$. Such a large bar angle would mean that the distance to the bar would be almost the same between $l=27^{\circ}$ and $l=5^{\circ}$ so the peak in Figure 4 would be more or less in the same position and not show the systematic change that is seen. The $75^{\circ}$ position angle was determined principally on star formation region the far end of the bar corresponding to a peak at $l=-22^{\circ}$. A $43^{\circ}$ bar angle would put the far end of the bar near $l=-12^{\circ}$ and indeed there is a peak in $C O B E$ $2.2 \mu \mathrm{m}$ surface brightness maps at this position (see Figs. 1 and 6 of Paper 1). It should be noted that the peak in the $C O B E 2.2 \mu \mathrm{m}$ surface brightness maps at $l=-22^{\circ}$ still needs to be explained and it is possible that there is a ringlike structure with tangential points at $l=27^{\circ}$ and $l=-22^{\circ}$, however a discussion of this is beyond the scope of this letter.
Many authors have examined the bulge off the plane and found that it is triaxial (e.g. Dwek 1995; Freudenreich 1998; López-Corredoira et al. 2000), however the position angle is small, typically about $15^{\circ}$. This is about $30^{\circ}$ different from the angle derived here for the bar. Although feature seen at $l=27^{\circ}$ cannot be the bulge as the latter does not give significant counts beyond about $l=12^{\circ}$, the bar apparently does runs into the bulge near $l=12^{\circ}$. Therefore, although geometrically the two features are distinct, currently it is not clear if the are dynamically linked and hence different aspects of the same phenomenon or where there are two independent bar-like features in the inner Galaxy, the triaxial bulge which dominates the inner few hundred pc and a major bar which extends to about $4 \mathrm{kpc}$.

\section{CONCLUSIONS}

There is a major old component in the $l=27^{\circ}$ line of sight at a distance of about $5.7 \mathrm{kpc}$ from the Sun. The component is seen at $l=20^{\circ}$ and $15^{\circ}$ and merges with the bulge inwards of this. The $l=27^{\circ}$ to $21^{\circ}$ region is already known to have a very high density of young stars and hence all of the expected properties of a bar with a position angle of around $43^{\circ}$ are present. The distribution of old stars is therefore very similar to that suggested for CO in Nakai (1992). The position angle is, however, significantly different from that of the triaxial bulge leading to the possibility that the Milky Way is a double-barred spiral galaxy.

\section{ACKNOWLEDGMENTS}

The TCS is operated on the island of Tenerife by the Instituto de Astrofísica de Canarias at the Spanish Observatorio del Teide of the Instituto de Astrofísica de Canarias. We would like to thank the referee for some valuable suggestions.

\section{REFERENCES}

Binney J., Gerhard O., Stark A., Bally J., Uchida K., 1991, MNRAS, 252, 210

Dwek et al. 1995, ApJ, 445, 716

Freudenreich H. T., 1998, ApJ, 492, 495

Hammersley P. L., Garzón F., Mahoney T., Calbet X., 1994, MNRAS, 269, 753 (Paper 1)

Garzón F.,López-Corredoira M., Hammersley P. L., Mahoney T.J, Calbet X., Beckman J.E., 1997, ApJ, 491, L31

López-Corredoira M., Hammersley P. L., Garzón F., Simonneau E., Mahoney T., 2000, MNRAS, 313, 392.

Nakai N., 1992 PASJ 44, L27

Peters W. L., 1975, ApJ, 195, 617

Reike G. H., Lebofsky M.J., 1985 ApJ, 288, 618

Sevenster M., Saha P., Valls-Gabaud D., Fux R., 1999, MNRAS 307,584

Wainscoat R. J., Cohen M., Volk K., Walker H. J., Schwartz D. E., 1992, ApJS, 83, 111 